\title{
Prayer-marks Heralding Acute Coronary Syndrome
}

Sir,

We read, with interest, the case report on extension of prayer-marks by Cangiano et al. and its association with worsening of the underlying chronic disease (1). We report a case where the occurrence of a similar skin lesion preceded an episode of acute coronary syndrome.

A 45-year old man presented with sudden onset of severe retrosternal chest pain radiating to the

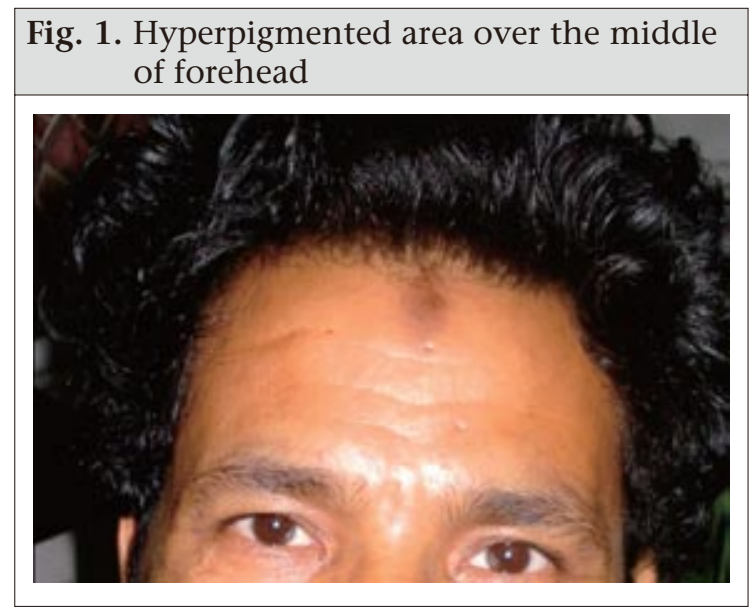

left arm, which was associated with sweating. His ECG revealed changes consistent with anterior wall myocardial infarction. Hyperpigmented areas over the middle of his forehead (Fig. 1) and knees (Fig. 2) had developed over the last two months. He was a Muslim by religion and regularly attended to his religious prayers (Namaaj), which involves kneeling and touching the ground with the forehead. Such skin changes, called Namaaj sign by us, have been described in earlier reports as a 'prayer sign' or 'prayer nodules' but no name has been assigned to the skin lesions $(2,3)$. These lesions have been

Correspondence and reprint requests should be addressed to:

Dr. Sourabh Aggarwal

Department of Medicine

University College of Medical Sciences

New Delhi 11095

India

Email: drsourabh79@gmail.com found to be associated with lichenification, acanthosis, basal cell hyperpigmentation, hyperkeratosis, hypergranulosis, and dermal papillary fibrosis (2).

Our report emphasizes the association of the prayer sign (Namaaj sign) with acute conditions in addition to chronic conditions as suggested by Cangiano et al. However, further studies are needed to

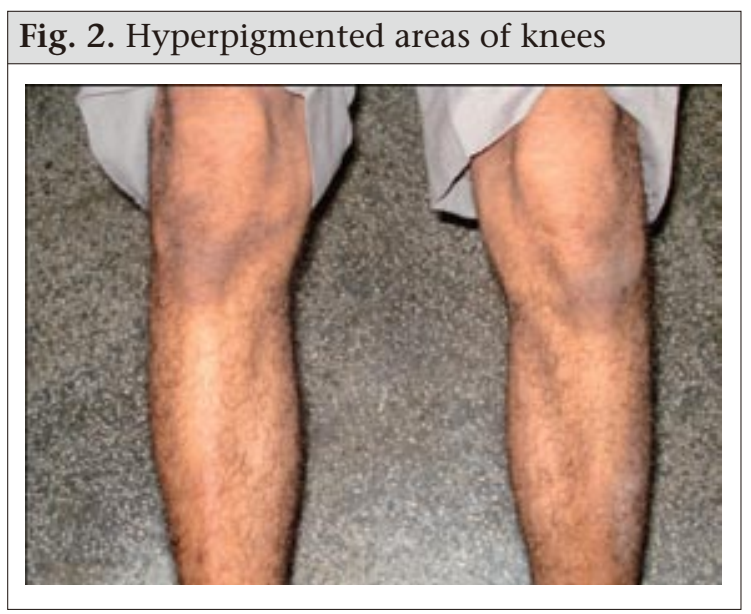

prove a statistically significant link between the two entities.

\section{ACKNOWLEDGEMENTS}

The authors thank Dr. Shridhar Dwivedi for introducing the term Namaaj sign to them.

\section{REFERENCES}

1. Cangiano M, Chisti MJ, Pietroni MAC, Smith JH. Extending prayer marks as a sign of worsening chronic disease (case study). J Health Popul Nutr 2011;29:290-1.

2. Abanmi AA, Al Zouman $\mathrm{AY}, \mathrm{Al}$ Hussaini $\mathrm{H}, \mathrm{Al}$-Asmari A. Prayer marks. Int J Dermatol 2002;41:411-4.

3. English JS, Fenton DA, Wilkinson JD. Prayer nodules. Clin Exp Dermatol 1984;9:97-8.

Vishal Sharma, Alka Sharma, and Sourabh Aggarwal Department of Medicine, University College of Medical Sciences, New Delhi 11095, India 